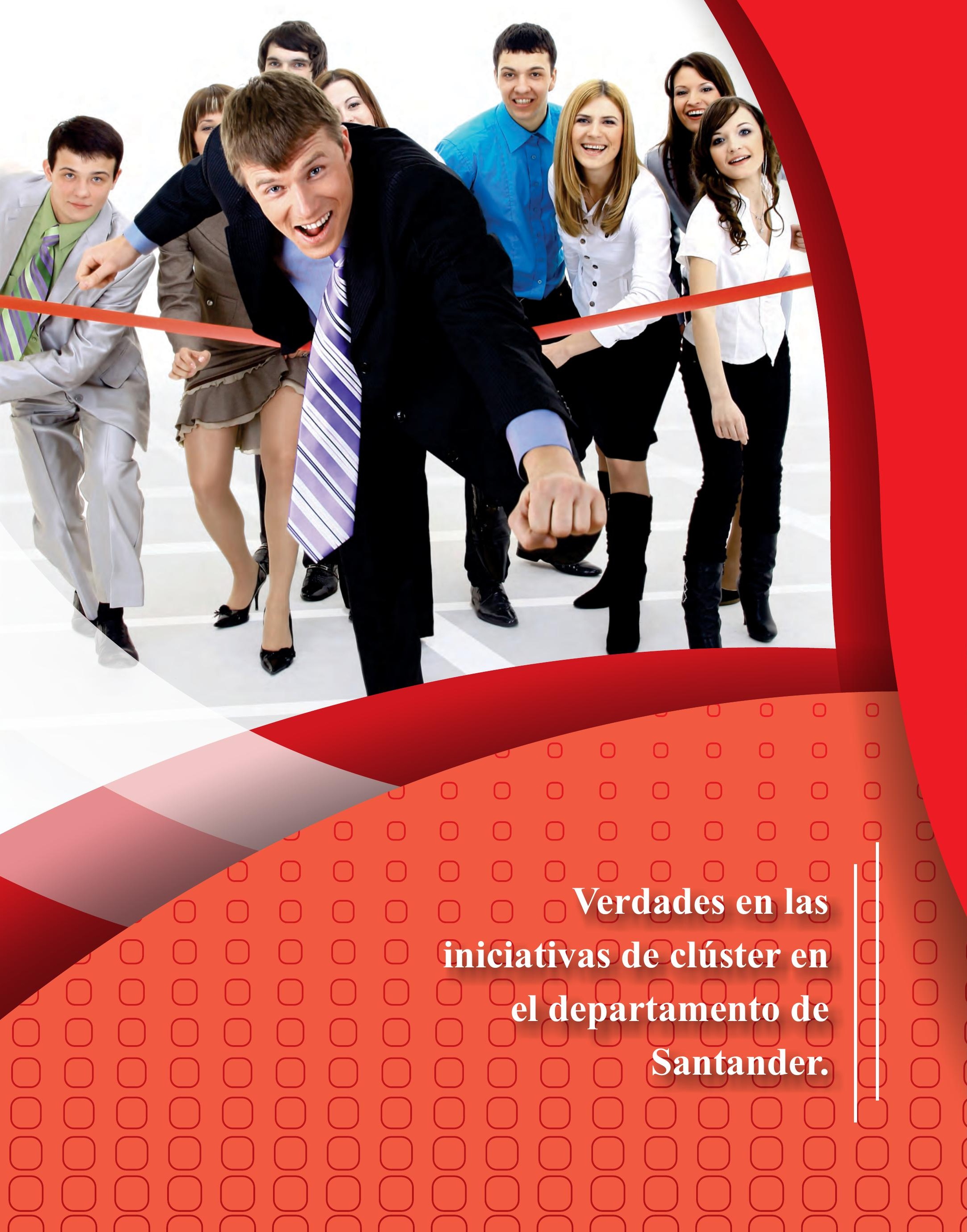




\title{
Verdades en las iniciativas de clúster en el departamento de Santander. ${ }^{1}$
}

\section{Facts in the Cluster Iniciatives in the Department of Santander.}

\author{
Oscar Alexander Manrique Salas. ${ }^{2}$ \\ ${ }^{1}$ Universidad Santo Tomás, Colombia.
}

Artículo recibido en el mes de Agosto de 2013; artículo aceptado en el mes de Octubre de 2013.

Citación del artículo: Manrique, O. (2013). Verdades en las iniciativas de clúster en el departamento de Santander. I+D Revista de Investigaciones, 2(2), 29-40.

\section{Resumen}

La implementación de sistemas de competitividad para el desarrollo económico en la región de Santander, ha sido de interés general, pero a su vez ha sido una iniciativa que ha dejado inmensos interrogantes respecto a su materialización, en virtud de diferentes factores que la hacen cada vez más difícil de concretar.

En el presente trabajo de investigación, se describe el proceso de la iniciativa clúster del petróleo y gas, calzado y metalmecánica, en el departamento de Santander, analizando su eficacia en la ejecución de sus objetivos estratégicos de competitividad de la región y su zona de influencia. Se describe cada uno de los aspectos deficitarios a partir de un análisis de fortalezas y debilidades por sector, respondiendo a la pregunta de investigación acerca de ¿Cuáles son las fortalezas, debilidades y características generales de los clústeres de cuero, manufactura, petróleo y gas y metalmecánico en la región de Santander?

Palabras clave: Clúster, metalmecánica, manufactura, calzado, petróleo, gas.

\section{Abstract}

The competitiveness systems implementation for the economic development in the region of the Department of Santander has been a general interest issue, but, at the same time, it has been an iniciative with many questions for coming it true, because of the several factors that complicate it.

In this work, the oil, gas, footwear and metallurgical industry clusters in the Department

\footnotetext{
${ }^{1}$ Artículo de investigación científica, de enfoque cuantitativo, resultado parcial de un proyecto de investigación en clústeres del departamento de Santander.

${ }^{2}$ Ingeniero Industrial, Universidad Industrial de Santander. Magíster en Administración de Empresas, Universidad Santo Tomás de Aquino. Correo electrónico: oscar_manrique@hotmail.com
} 
of Santander are described and analyzed in their efficacy to execute their strategical competitiveness objectives in the region and their influence zone. Every sector lacking aspects are commented, using a strengths and weaknesses analysis scheme, responding to the question about ¿What are strengths, weaknesses and general characteristics in the Department of Santander, the oil, gas, footwear and metallurgical industry clusters?

Keywords: Cluster, metallurgical industry, manufacture, footwear, oil, gas.

\section{Introducción}

Los clústeres son hoy en el mundo una estrategia importante de promoción del desarrollo económico y de la competitividad. Los clústeres son consolidaciones de diversos sectores económicos y productivos de una región, que de una u otra manera aportan grandes beneficios a las economías de escala, al lograr y aceptar a los sectores y empresas más pequeñas y de mediana estructura, funcionar de manera agrupada con algunas ventajas que caracterizan a las empresas de mayor tamaño, manteniendo su flexibilidad, autonomía y competitividad (Dirven, 2006). Se ha evidenciado, de acuerdo con las experiencias obtenidas alrededor del mundo, que existen factores determinantes para obtener el éxito competitivo, entre los cuales se encuentran la proximidad espacial y sociocultural de los elementos de los clúster; dichos factores facilitan la creación y la difusión de innovaciones, favoreciendo la existencia de mercados laborales especializados (López, 2006).
Entre las iniciativas de clúster más exitosas, se encuentran las de Calgary (Canadá), Houston (U.S.A) y Mar del Norte (Reino Unido y Noruega). Según el Índice de Competitividad Global del Foro Económico Mundial (WEF, 2010), Colombia ocupa el puesto 69 entre 131 países, lo cual sugeriría que se hace necesario introducir una política de competitividad nacional (DNP, 2009) sobre la cual se deben diseñar las estrategias para el desarrollo en empresas, municipios y otros entes gubernamentales (Conpes, 2008).

La formación de clústeres en Santander entre 2007-2010, ha sido una estrategia anhelada por sus implicaciones para la competitividad y para el desarrollo. A partir del año 2010, al interior de la iniciativa clúster del petróleo y gas de Santander, se redefinieron los objetivos estratégicos (Ramírez \& Cuéllar, 2008) a partir de las reflexiones emanadas de la firma Clúster Consulting, la cual ha implementado estrategias para iniciativas clúster en Latinoamérica (Monge, 2002), a instancias de su convocatoria por la Comisión Regional de Competitividad y la Cámara de Comercio de Bucaramanga. A partir de ello se ha planteado una iniciativa nueva hacia el 2020 (Plata, 2010), con convenios vigentes de cooperación con países como Corea, Estados Unidos y Francia (Comité Técnico, 2010); sin embargo, los empresarios en el sector no han entendido la magnitud de la iniciativa clúster y que su visión hacia la competitividad es a corto plazo; no existe confianza entre ellos para trabajar de manera asociativa, por la falta de una política clara y gobernabilidad; se hacen esfuerzos individuales por conseguir y avanzar en aspectos de competitividad; se han realizado visitas empresariales internacionales, pero las 
propuestas recomendadas en ellas han quedado olvidadas por el temor de arriesgarse al emprendimiento de algo nuevo y revolucionario en materia de centros industriales (CRC, 2008).

Se ha ganado espacio en la formación de competencias técnicas y humanas a través del Servicio Nacional de Aprendizaje (SENA), quien ha establecido convenios y promovido los ambientes tecnológicos competitivos a través del mantenimiento electromecánico, mecanizado, eléctrico, operarios de plantas industriales; en las áreas transversales como la Química Industrial, Electricidad y Sector Metalmecánico se han establecido convenios con el Ministerio de Educación Francés y el Instituto Schneider, para iniciar el proceso de transformación metodológica y tecnológica; con relación al cuerpo docente, se han venido preparando a nivel nacional e internacional y poseen el Centro de Control Numérico Computarizado.

En el presente trabajo de investigación, se describe el proceso de la iniciativa clúster del petróleo y gas, calzado y metalmecánica en el departamento de Santander, analizando su eficacia en la ejecución de sus objetivos estratégicos de competitividad de la región y su zona de influencia.

\section{Método}

Se llevó a cabo una investigación no experimental descriptiva-transversal, para obtener datos relacionados con las variables de los clústeres en estudio.

\section{Participantes}

Empresarios, dirigentes, expertos y personas asociadas con el sector metalmecánico, del calzado, la manufactura, el petróleo, el gas en el departamento de Santander.

\section{Materiales e instrumentos entrevistas estructuradas.}

A través de las entrevistas, se recogió información relacionada con aspectos de innovación, asociatividad, gobernabilidad, sinergia, competitividad, desarrollo, de cada uno de los sectores implicados.

\section{análisis documental.}

A través de las revisiones de documentos, publicaciones, eventos del sector público y privado, nacional e internacional, vinculados con la competitividad y el desarrollo.

\section{matriz DOFA.}

A través de análisis de los hallazgos encontrados y triangulación de información de cada sector estudiado.

\section{Procedimiento}

Se llevó a cabo una revisión de antecedentes nacionales, internacionales y regionales, sobre las estrategias e iniciativas de clúster, sus resultados, desarrollos, avances y deficiencias a la fecha.

Se recogió información sobre entes regionales y locales del sector público, acerca del manejo de la competitividad en el departamento y sus gestiones al respecto. 
Se analizaron diferentes eventos académicos relacionados con iniciativas de clúster, para estudiar tendencias en los sectores y en la misma iniciativa.

Se llevó a cabo la recolección de información mediante encuestas y entrevistas con diferentes participantes, en los diferentes sectores económicos y empresariales.

\section{Resultados}

Se presentan a continuación los resultados que describen el estatus de desarrollo de las iniciativas de clúster en algunos renglones de la economía del departamento, considerados estratégicos.
Estatus de efectividad y progreso del clúster de petróleo y gas en el departamento deSantander.

La exploración realizada en el clúster de petróleo y gas en el departamento de Santander, arrojó los resultados desglosados a continuación.

Aplicando la matriz del cuadrante de efectividad, se interrelacionó a los objetivos estratégicos vs el progreso de la implementación del clúster en petróleo y gas del departamento, encontrando que con respecto a los primeros, se obtuvo un valor porcentual de $42,66 \%$, indicando un estatus de ninguna implementación, y con respecto al progreso en la implementación, se obtuvo un porcentaje de $62 \%$, es decir, un nivel insatisfactorio, lo cual se presenta en la tabla 1 .

Tabla1. Resultado de la matriz del cuadrante de efectividad para la IC

\begin{tabular}{|c|c|c|c|c|}
\hline & & \multicolumn{3}{|c|}{$\begin{array}{l}\text { OBJETIVOS } \\
\text { ESTRATEGICOS }\end{array}$} \\
\hline \multirow{5}{*}{$\begin{array}{c}\text { PROGRESO } \\
\text { DE LA } \\
\text { IMPLEMENTACIÓN }\end{array}$} & & 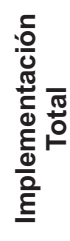 & 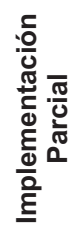 & 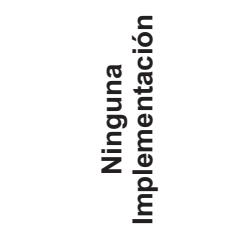 \\
\hline & Muy Satisfactorio & & & \\
\hline & Satisfactorio & & & \\
\hline & Insatisfactorio & & & $\begin{array}{c}\text { Iniciativa Clúster } \\
\text { Petróleo y Gas de } \\
\text { Santander }\end{array}$ \\
\hline & Muy Insatisfactorio & & & \\
\hline
\end{tabular}

Cuadro 1. En color, ubicación de la iniciativa clúster respecto a los objetivos estratégicos y progreso de la implementación. Fuente: Autor.

De acuerdo con los aspectos evaluados al interior de estos hallazgos, se encontró que existían áreas deficitarias y de aseguramiento en los clústeres de petróleo y gas del departamento de Santander, en las categorías de innovación, asociación, gobernabilidad y direccionamiento, los cuales denotan diferentes características que hacen difícil la consolidación y maduración del mismo, tal como se aprecia en la tabla 2. 
Tabla 2. Carencias halladas respecto al clúster de petróleo y gas en el Departamento de Santander.

\begin{tabular}{|c|c|c|}
\hline Categoría & Descripción de carencias & Aspectos a asegurar \\
\hline Innovación & $\begin{array}{l}\text { Sinergia entre la industria y la academia } \\
\text { para la investigación y en la asignación de } \\
\text { recursos para la I\&D. }\end{array}$ & $\begin{array}{l}\text { Infraestructura de centros de } \\
\text { capacitación técnica, procesos de } \\
\text { mejoramiento por competencia } \\
\text { empresarial. }\end{array}$ \\
\hline Asociación & $\begin{array}{l}\text { Desconfianza entre las empresas para } \\
\text { integrar la iniciativa, la interpretación } \\
\text { errónea de la filosofía de clúster, los } \\
\text { intereses por la búsqueda de negocios e } \\
\text { intereses particulares. }\end{array}$ & $\begin{array}{l}\text { Conformación de redes de negocios a } \\
\text { nivel nacional e internacional. }\end{array}$ \\
\hline Gobernabilidad & $\begin{array}{l}\text { Política de apoyo a los clúster, bajo } \\
\text { liderazgo y politización. }\end{array}$ & $\begin{array}{l}\text { Reorientación y definición de acuerdos } \\
\text { y trabajo en conjunto con los } \\
\text { participantes. }\end{array}$ \\
\hline Sector privado & $\begin{array}{l}\text { Cabeza visible del clúster, búsqueda de } \\
\text { intereses particulares sobre los colectivos, } \\
\text { entre ellos los de la Cámara de Comercio y } \\
\text { empresarios. }\end{array}$ & $\begin{array}{l}\text { Generación de confianza de inversión } \\
\text { extranjera en la zona y captación de } \\
\text { recursos de diferentes niveles de } \\
\text { producción. }\end{array}$ \\
\hline
\end{tabular}

Fuente: Autor.

\section{Estado de madurez de la iniciativa clúster de Santander}

De acuerdo con los resultados obtenidos y al análisis de la literatura relacionada con las iniciativas clúster, podemos concluir que la iniciativa clúster del petróleo y gas del departamento de Santander se encuentra en la etapa emergente, en atención al bajo compromiso, la escasa sinergia y poca comprensión del clúster y sus beneficios, las escasas políticas para su apoyo, entre otros factores más, que se representan gráficamente en la figura 1 . 
Figura 1. Estado en el ciclo de vida de la iniciativa de clúster del petróleo y gas de Santander. Se localiza el estatus emergente de madurez del clúster del petróleo y del gas en Santander en el sector indicado por la flecha.

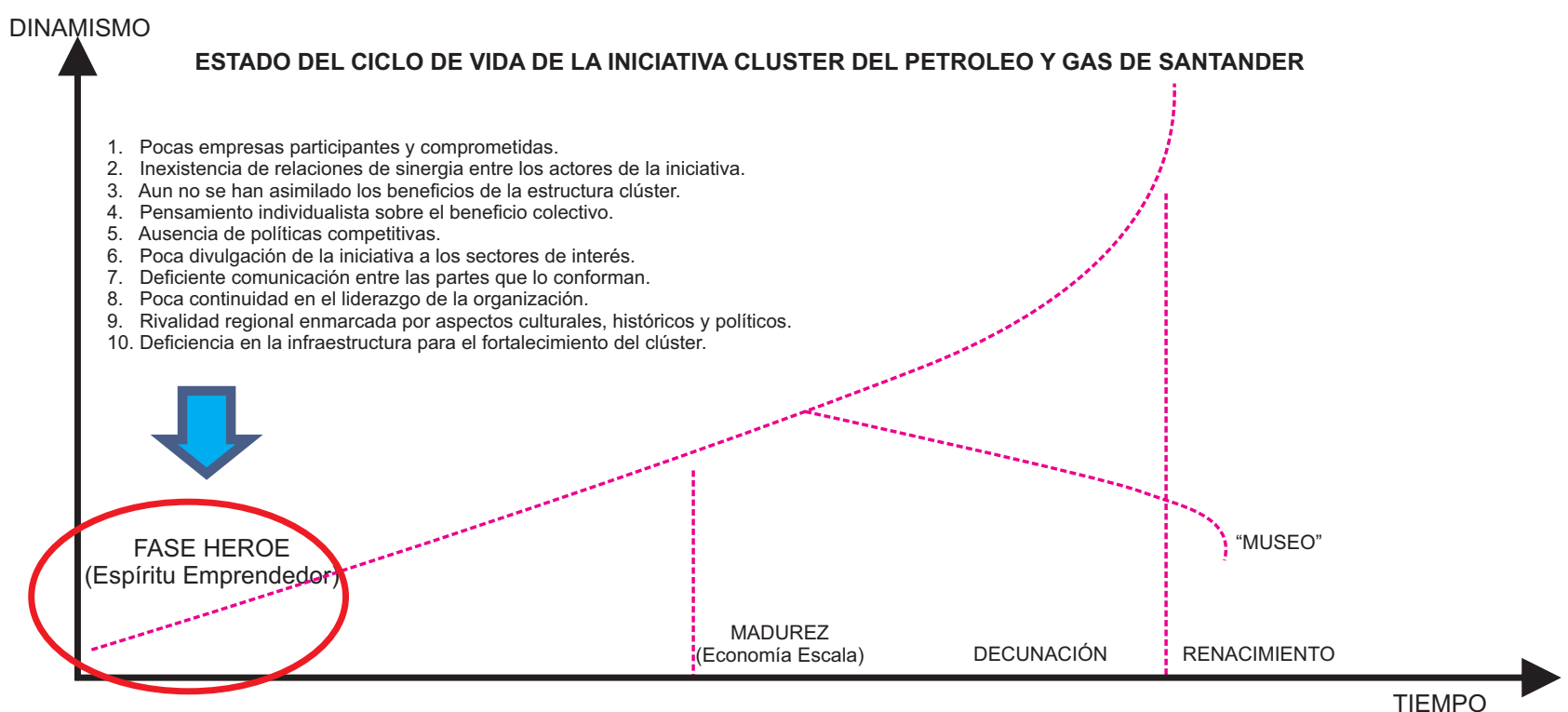

Fuente: Elaboración del autor basándose en Clúster Equilibrando Fuerzas Evolutivas y Constructivas. Orjan Sólvell (2009)

\section{Estatus de efectividad y progreso del clúster de cuero en la ciudad de Bucaramanga.}

En la figura 2 se propone un diagrama estructural para el clúster de la cadena del cuero, calzado y marroquinería en el departamento de

complementario como el de las confecciones, del cual ya se dispone en Santander; el sector de

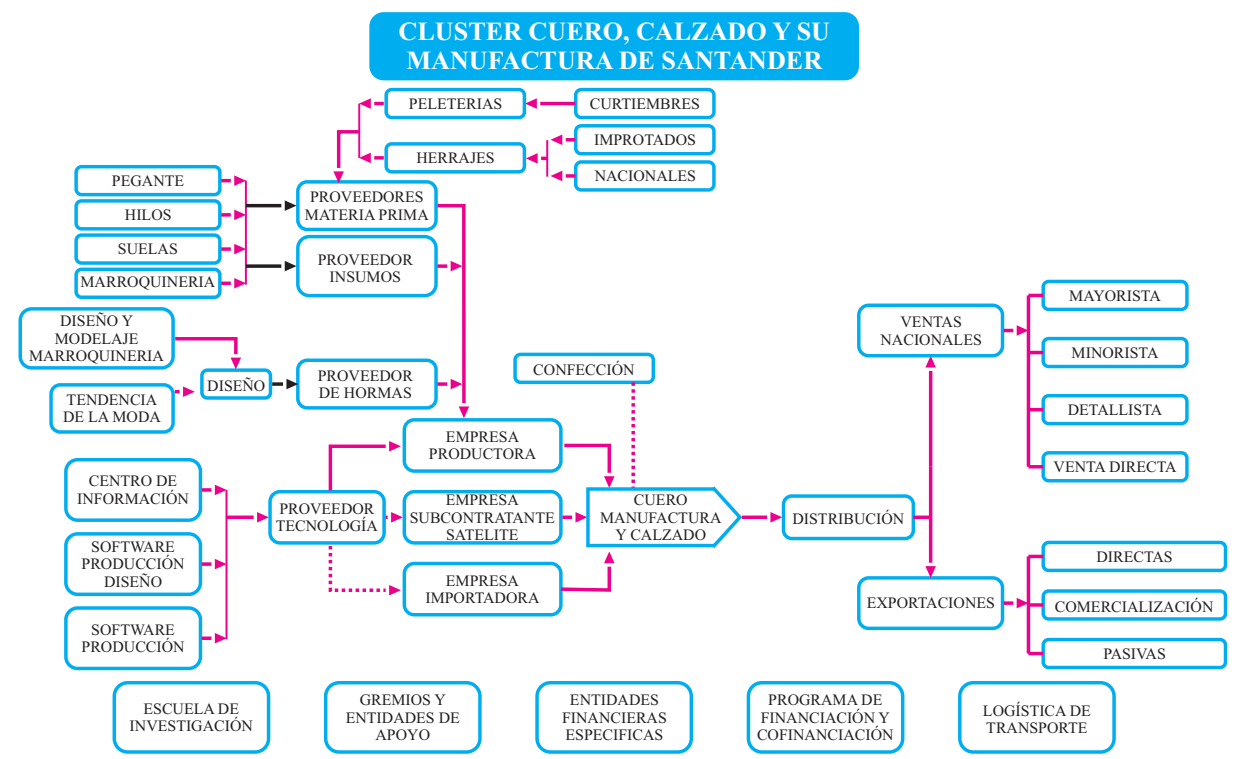

Fuente: Modelo expuesto por Moreno, 2008, pág. 16 
software, el cual, aunque existen empresas y capacidades de recurso humano para generarlos, están dedicados a la producción de software contable y no han desarrollado o adaptado los programas para producción y diseño como lo propone el esquema planteado.

(b) Las fortalezas en diseño no son las más apropiadas, aunque la institución ITAE ha desarrollado el programa de diseño de modas, realizando ferias y desfiles para impulsar este concepto, con algunos avances preliminares; adicionalmente en este año 2010, el gobierno municipal ha iniciado el proceso para la construcción de un centro de diseño que apoye no solo la cadena del cuero, sino otras como las confecciones, las artes gráficas y la joyería.

(c) En cuanto a los proveedores, vincula o clasifica aquellos que suministran partes para la fabricación del calzado u hormas, aquellos que suministran insumos como las suelas, los hilos, el pegante, y los proveedores del cuero y herrajes, que a su vez dependen de las curtiembres nacionales e internacionales; en este rubro de proveedores se ha trabajado con pasividad pero con resultados en el tema del cuero con avances poco notables; no hay trabajo alrededor de los precios de las materias primas ni del control a su calidad.

(d) En cuanto a proveedores de tecnología, se cuenta con Ceinnova como centro de investigación, el cual, a pesar de su disponibilidad no tiene el impacto esperado, explicación que no está al alcance de este informe.

(e) Dentro del eslabón central de la cadena, encontramos a los actores que se clasifican en importadores del producto, productores $y$ contratistas (ya sea como maquiladoras o simples tercerizadores) incluidos en el clúster, pero la relación apropiada o sinérgica entre las mismas no es clara, o falta una exposición en prosa para explicarla.

(f) También se identifican los eslabones de distribución, con sus canales de venta directa, detallista, minorista y mayorista dentro de lo nacional, y los canales de distribuidores (comercialización), venta pasiva (es decir, por pedido de un cliente foráneo) y la venta directa donde se realiza el mercadeo para obtener el cliente.

(g) Aparecen otras entidades afines que se describen como: i) escuelas de investigación, de las cuales se pueden encontrar los $C D P y$ Ceinnova, unido a los grupos de investigación en diseño de ITAE, y de productividad y competitividad de la Universidad Cooperativa de Colombia (UCC) y la Universidad de Santander (UDES); también instituciones o entidades de agremiación, de las cuales se cuenta con la Asociación Colombiana de Industriales del Calzado, el Cuero y sus Manufacturas (ACICAM), con un objetivo de desarrollo productivo, la Asociación de Industriales del Calzado y Similares (ASOINDUCAL'S) con un objetivo de incentivo o fomento de la comercialización a través de ferias inicialmente, la Asociación de Industriales del Calzado de Floridablanca (ASICAF) y la Asociación de Peleteros de Colombia (ASPECOL); por otra parte, las entidades financieras y los programas de financiamiento, disponen de un amplio portafolio al respecto entre el sector financiero 
privado y las instituciones de apoyo a las MiPymes (Bancoldex, Fogafin), pero a las que se limita su acceso por la misma indisposición financiera de las empresas; finalmente, el sector del transporte, el cual es un apoyo en materia de efectividad, pero que aún requiere mejoras en los costos, los cuales dependen del mejoramiento de la infraestructura vial nacional.

\section{Estatus de efectividad y progreso del} clúster de metalmecánica en la ciudad de

\section{Bucaramanga}

En este clúster se halló que las empresas buscan nuevos negocios; esa es la motivación fundamental, y secundariamente, el incremento en la productividad y en la competitividad. Una de las principales debilidades es la formación del personal. Las empresas consideran que la formalización empresarial es la condición más

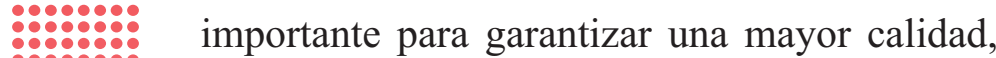

productividad y competitividad a lo largo de las cadenas. De igual manera se identifica que las certificaciones en aspectos de calidad son prerrequisitos más que elementos diferenciadores. Otras debilidades halladas fueron la baja asociatividad, ausencia de propuestas concretas y de divulgación, baja credibilidad y continuidad, poco tiempo disponible, monopolios, mal manejo de recursos, desmotivación.

La figura 3 presenta resultados del análisis DOFA al sector Metalmecánico de Santander, adelantado por la ANDI Santanderes - Grupo $10^{\mathrm{M}}$, y en el cual se establece una serie importante de elementos claves para el desarrollo de este sector en la región.

Figura 3.DOFA al sector metalmecánico de Santander.

\section{Discusión}

A partir de los resultados obtenidos, se aprecia que algunos de los actores o líderes en los diferentes

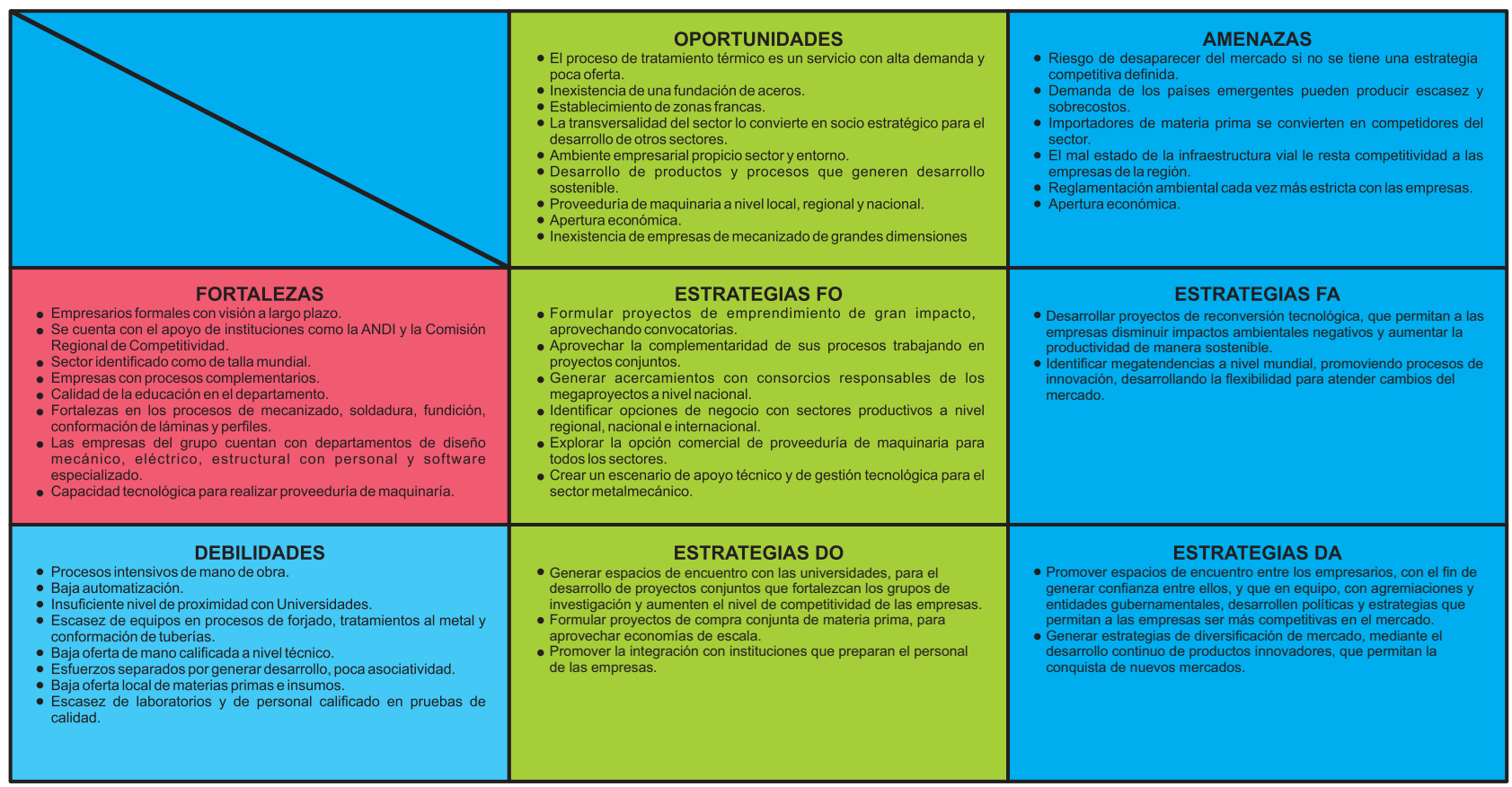

Fuente: Primer informe Proyecto-Metalmecánico de Santander año 2011 - ANDI - Santanderes - Grupo $10^{\circ}$ 
sectores evaluados muestran poco compromiso, voluntad y gestión, respecto de las estrategias de competitividad para el desarrollo del clúster, lo cual no ha permitido avanzar en el cumplimiento de las acciones inmersas en los objetivos estratégicos; para ello se requiere definir y precisar las funciones y responsabili-dades de cada uno de los actores involucrados en el proceso. Por otra parte, la iniciativa clúster no ha sido visualizada como eje primordial para el desarrollo y crecimiento empresarial de la región, tampoco al interior de la iniciativa clúster se ha definido con exactitud la identificación del negocio; los empresarios inscritos como participantes en la iniciativa clúster no se han involucrado de lleno en el proceso, no tienen muy claras las ventajas competitivas de la filosofía clúster.

Paralelamente, puede considerarse a partir de los hallazgos del estudio, que la iniciativa clúster carece de un sistema de monitoreo de evaluación de desempeño, en el cual se involucre a sus actores e indicadores de competitividad, que reflejen el avance del mismo en la ejecución de los objetivos estratégicos y la materialización de las realizaciones cumplidas. Se requiere por lo tanto más asociatividad; la coordinación y cooperación entre los diferentes actores de la iniciativa clúster debe ser fluida, ágil y eficiente, de tal manera que se permita avanzar en los objetivos estratégicos y metas de la misma. En la iniciativa clúster no se han considerado ni valorado los casos exitosos y experiencia de las Cámaras de Comercio a nivel nacional, en otras iniciativas clúster (Medellín Ciudad Clúster y Clúster del Turismo del Eje Cafetero), las cuales a través de su trabajo en equipo, han impactado satisfactoriamente en sectores económicos, beneficiando las regiones a las cuales pertenecen. Dentro de las experiencias exitosas a nivel mundial, se ha considerado como buena estrategia la incorporación de empresas multinacionales al interior de la estructura de la iniciativa clúster, ya que esto incentiva y promociona a las empresas de la región a transformar, invertir y adoptar medidas de clase mundial en sus procesos.

Por otra parte, la Dirección Ejecutiva de la iniciativa clúster debe ser visible y estable en el tiempo, de tal manera que tenga poder de convocatoria y estimule al seguimiento de los objetivos estratégicos, así como también sea capaz de garantizar la cohesión grupal. La Dirección Ejecutiva de la iniciativa, debe estar rodeada de personas que crean y apoyen en el proyecto al cual pertenecen, sin distingo alguno de creencias, aspectos políticos o intereses particulares. La Dirección Ejecutiva debe incentivar entre los miembros de la iniciativa clúster una excelente actitud, compromiso, participación, mentalidad abierta, voluntad y responsabilidad, de tal manera que se garantice y permanezca en el tiempo la motivación por el proceso al que pertenecen. Se debe estructurar un grupo de entidades que lideren la iniciativa clúster y otras con las que se realicen alianzas estratégicas que apoyen; así mismo se debe buscar asesorías en entidades como la RedLAC (Red Latinoamericana de Clúster) y otras iniciativas clúster exitosas a nivel nacional e internacional.

Con respecto al logro, una Dirección sola no basta para lograr y conseguir los resultados esperados en el tiempo, para ello se requiere de una disciplina organizacional del equipo de 
trabajo, basada en la consecución de los objetivos estratégicos, a través de la redistribución de responsabilidades y asignación de tareas; para ello se hace necesario efectuar seguimiento estricto a los roles, responsabilidades, avances y barreras presentadas, con el fin de garantizar el cumplimiento del plan estratégico de la iniciativa. Para ello se hace necesario y prioritario definir y clasificar el grado de impacto de cada uno de los objetivos y acciones estratégicas, asegurando de manera preliminar, el diseño de un sistema de gestión, basado en unos indicadores de desempeño retadores en el tiempo, y con los cuales se realice la medición y seguimiento de los mismos, bajo la estructura de un cuadro de gestión y control. El proceso de la iniciativa clúster, como muchos otros, requiere de inversiones de recursos destinados para las mejoras en la red de infraestructura, promoción internacional, investigación y desarrollo, con los cuales se debe garantizar el inicio y continuidad de las actividades encaminadas a lograr la consecución y cumplimiento de los objetivos estratégicos de la iniciativa clúster.

Finalmente, al interior de la iniciativa clúster no se han desarrollado líneas o redes de investigación, y el número de proyectos sobre la temática clúster es reducido, en comparación con todas las oportunidades existentes para cada uno de los frentes de trabajo en los cuales dichas investigaciones podrían generar grandes beneficios y oportunidades para la región; para ello, las universidades y entes académicos deben incentivar y orientar la investigación, innovación y desarrollo a través de foros, seminarios, congresos, semilleros. Así mismo se deben garantizar los recursos económicos para la apertura de nuevos programas que requiera la región, la firma de convenios con universidades internacionales buscando la internacionalización de la educación, elevar la calidad de la misma y potenciar el recurso humano calificado.

Las universidades deben tener un protagonismo más activo en la dinámica investigativa de la iniciativa clúster en Santander, que contribuya de manera efectiva al fortalecimiento de las capacidades técnicas, humanas e intelectuales del recurso humano de la región y con el cual se impacten de manera positiva las oportunidades de negocio, suplan las necesidades de los diferentes sectores y promuevan la competitividad de la región.

\section{Referencias}

Comisión Regional de Competitividad (Diciembre, 2008). Informe final Plan Regional de Competitividad de Santander.

Comité Técnico Clúster de Petróleo y Gas (2010). Priorización de acciones 2010. Documento borrador.

Consejo Nacional de Política Económica y Social (2008). Política Nacional de Competitividad y Productividad (No 3527). Bogotá.

Departamento Nacional de Planeación (2009). Así va la Politica Nacional de Competitividad(Documento No 3). Bogotá.

Dirven, M. (2006). Acción conjunta en los clústers: entre la teoría y los estudios de caso 1. Recuperado de CEPAL

http://www.fidamerica.org/admin/docdescargas/cent 
rodoc/centrodoc_401.pdf

López, A., Hernández, J. (2006). Vías para la competitividad. Revista DYNA, 150.

Monge, G. (2002). Promoción del clúster en América Latina: La experiencia del Centro Latinoamericano para la Competitividad y el Desarrollo Sostenible (CLACDS) del INCAE.

Plata, O. (2010). Clúster de petróleo y gas: Proceso de Iniciativas de Desarrollo. Bucaramanga.

Ramírez, J. \& Cuéllar, E. (2008). Clúster del petróleo y del gas, evaluación del entorno competitivo, agenda estratégica y elementos de una Iniciativa de Desarrollo del Clúster. Bogotá: Centro de Estrategia y Competitividad: Universidad de los Andes.

World Economic Forum (2010-2011). The global competitiveness report. 